\title{
Proportional-Integral Projected Gradient Method for Model Predictive Control
}

\author{
Yue Yu, Purnanand Elango, and Behçet Açıkmeşe
}

\begin{abstract}
Recently there has been an increasing interest in primal-dual methods for model predictive control (MPC), which require minimizing the (augmented) Lagrangian at each iteration. We propose a novel first order primal-dual method, termed proportional-integral projected gradient method, for MPC where the underlying finite horizon optimal control problem has both state and input constraints. Instead of minimizing the (augmented) Lagrangian, each iteration of our method only computes a single projection onto the state and input constraint set. Our method ensures that, along a sequence of averaged iterates, both the distance to optimum and the constraint violation converge to zero at a rate of $O(1 / k)$ if the objective function is convex, where $k$ is the iteration number. If the objective function is strongly convex, this rate can be improved to $\mathrm{O}\left(1 / \mathrm{k}^{2}\right)$ for the distance to optimum and $\mathrm{O}\left(1 / \mathrm{k}^{3}\right)$ for the constraint violation. We compare our method against existing methods via a trajectory-planning example with convexified keep-out-zone constraints.
\end{abstract}

Index Terms-Model predictive control, optimization algorithms

\section{INTRODUCTION}

$\mathbf{M}$ ODEL predictive control (MPC) provides a systematic approach for automatic control with physical and operational constraints [1]-[5]. The key ingredient in MPC is solving a finite horizon discrete-time convex optimal control problem that can be expressed in the following form

$$
\begin{array}{ll}
\underset{z}{\operatorname{minimize}} & \frac{1}{2} z^{\top} H z+h^{\top} z \\
\text { subject to } & G z=g, \quad z \in \mathbb{Z},
\end{array}
$$

where the trajectory variable $z$ aims to minimize a convex quadratic cost function $\frac{1}{2} z^{\top} H z+h^{\top} z$ subject to linear dynamics constraints $G z=g$ together with convex state and input constraint $z \in \mathbb{Z}$. Throughout we assume $\mathbb{Z}$ is the Cartesian product of convex sets whose Euclidean projection can be evaluated at low computational cost. Such an assumption applies to many practical state and input constraints in MPC [6]-[9]; see Tab. I] and [10] for some examples. In addition to convex MPC problems, solution to problem (1) is also an integral part of problems with nonlinear dynamics and nonconvex constraints. In these cases, a sequence of convex subproblems modeled by (1) are solved to obtain the solution

Accepted to IEEE Control System Letters, available at https: //ieeexplore.ieee.org/document/9295329 DOI: 10.1109/LCSYS.2020.3044977

The authors are with the Department of Aeronautics and Astronautics, University of Washington, Seattle, WA 98195 USA (emails: yueyu@uw.edu,pelango@uw.edu,behcet@uw.edu). of the original non-convex problem, as done in sequential convex programming [11], [12] and successive convexification methods [13]-[15].

TABLE I: Examples of simple convex sets and projections

\begin{tabular}{|c|c|}
\hline Set $\mathbb{X}$ & Projection of $x$ onto $\mathbb{X}$ if $x \notin \mathbb{X}$ \\
\hline$\left\{x \mid\|x\|_{2} \leqslant \alpha\right\}$ & $\frac{\alpha}{\|x\|_{2}}$ \\
\hline$\{x \mid l \leqslant x \leqslant u\}$ & $\min \{\max \{x, l\}, u\}$ \\
\hline$\{x \mid\langle a, x\rangle \leqslant \alpha, a \neq 0\}$ & $x-(\langle a, x\rangle-\alpha) \frac{a}{\|a\|_{2}}$ \\
\hline$\left\{x=(y, \alpha) \mid\|y\|_{2} \leqslant \alpha\right\}$ & $\frac{\left.\|y\|_{2}+\alpha\right) \text { if }\|y\|_{2} \leqslant-\alpha ;}{2\|y\|_{2}}\left(y,\|y\|_{2}\right)$ otherwise \\
\hline$\{x=(y, \alpha) \mid f(y) \leqslant \alpha\}$ & $x$ solves $y \in x+(f(x))$ where \\
\hline$\{x \mid f(x) \leqslant \alpha\}$ & $(I+\mu \partial f)^{-1}(x)$ where $f(x)$ \\
\hline & $\mu$ solves $f\left((I+\mu \partial f)^{-1}(x)\right)=\alpha$ \\
\hline
\end{tabular}

Here function $f: \mathbb{R}^{n} \rightarrow \mathbb{R}$ is continuous, convex and finite valued, $\partial f$ denotes the subdifferential of $f, \max / \mathrm{min}$ is evaluated element-wise.

Recently there has been an increasing interest in first order primal-dual methods for MPC. Such methods solve problem (1) together with its dual problem by updating both primal and dual variables at each iteration. For example, the dual fast gradient method first updates the primal variables by optimizing the Lagrangian, then the dual variables using Nesterov's method [8], [16], [17]. Similarly, the Chambolle \& Pock method first updates the primal variables by optimizing the augmented Lagrangian, then the dual variables using gradient ascent with extrapolation [18]. The alternating directional method of multipliers (ADMM) first updates two copies of the primal variables by optimizing the augmented Lagrangian: one subject to $G z=g$, and the other subject to $z \in \mathbb{Z}$. The dual variables then simply integrate the difference between the two copies [9], [19]-[21]. Compared with second order methods [22] and first order primal methods [7], first order primaldual methods allow both efficient per-iteration computation and general state and input constraints.

The common challenge in implementing the aforementioned primal-dual methods is optimizing the (augmented) Lagrangian during each iteration. In general, such optimization requires either inner loop iterations that costs multiple projections onto set $\mathbb{Z}$ [23], or the solution to system of linear equations that demands Ricatti recursion [20], [24] or precomputing matrix inverse/decomposition [9], [17], [19].

To address this challenge, several primal-dual methods have been developed recently, where each iteration only computes a single projection onto set $\mathbb{Z}$, rather than minimizing the augmented Lagrangian [25]-[27]. However, these results still have their limitations. In particular, no convergence rate was 
proved in [25]. On the other hand, while [26, Thm. 1, Thm. 4] and [27, Thm. 1] proved the convergence rate of a nonnegative duality gap function along a sequence of averaged iterates (also known as ergodic convergence), they however provide no convergence rate for the affine constraint violation, which is commonly used as a stopping criterion [16].

We propose a novel primal-dual method for MPC problem (1), termed proportional-integral projected gradient method, that also computes a single projection onto set $\mathbb{Z}$ per iteration. Our method ensures that, along a sequence of averaged iterates, both the distance to optimum and the constraint violation converges to zero at a rate of $O(1 / k)$ if the objective function is convex, where $k$ is the iteration number. If the objective function is strongly convex, this rate can be improved to $O\left(1 / k^{2}\right)$ for the distance to optimum and $O\left(1 / k^{3}\right)$ for the constraint violation.

The rest of the paper is organized as follows. After reviewing some related work in Section II] Section III introduces our method together with its convergence guarantee. Section IV discusses the implementation of our method on a tracking problem common in MPC. Section V demonstrates our results via a trajectory-planning example. Finally, Section VI concludes and comments on future directions.

Notation: Let $z, y \in \mathbb{R}^{n}$ and $H, P \in \mathbb{R}^{n \times n}$ with $H=$ $H^{\top}, P=P^{\top}$. We denote $\langle z, y\rangle=z^{\top} y,\|z\|_{2}=\sqrt{z^{\top} z}$, $\|z\|_{H}=\sqrt{z^{\top} H z}$. We say $H<(\leq) P$ if and only if $P-H$ is positive (semi-)definite. The Euclidean projection onto a closed convex set $\mathbb{Z} \subset \mathbb{R}^{n}$ is denoted by $\pi_{\mathbb{Z}}: \mathbb{R}^{n} \rightarrow \mathbb{Z}$ where

$$
\pi_{\mathbb{Z}}[z]=\underset{z^{\prime} \in \mathbb{Z}}{\operatorname{argmin}}\left\|z^{\prime}-z\right\|_{2} .
$$

\section{ReLATED WORK}

In this section, we briefly review some existing first order primal-dual methods for MPC. In the following, we let $k$ and $j$ denote iteration counters, and $\alpha$ the step size.

\section{A. Dual fast gradient method}

Assuming $H>0$ in (1), dual fast gradient method [8], [16], [17] solves problem (1) as follows

$$
\begin{aligned}
z^{k+1} & =\underset{z \in \mathbb{Z}}{\operatorname{argmin}} \frac{1}{2} z^{\top} H z+h^{\top} z+\left\langle v^{k}, G z\right\rangle, \\
w^{k+1} & =v^{k}+\alpha\left(G z^{k+1}-g\right), \\
v^{k+1} & =w^{k+1}+\frac{k}{k+3}\left(w^{k+1}-w^{k}\right) .
\end{aligned}
$$

The idea is to apply Nesterov's method [28, Sec. 2.2] to the dual problem of (11). In general, the minimization step in 2a can only be solved approximately using another inner loop of Nesterov's method [23], which iterates as follows [28, Sec. 2.2] ( $j$ denotes the inner loop iteration counter)

$$
\begin{aligned}
& z^{j+1}=\pi_{\mathbb{Z}}\left[y^{j}-\frac{1}{\lambda}\left(H y^{j}+h+G^{\top} v^{k}\right)\right], \\
& y^{j+1}=z^{j+1}+\frac{\sqrt{\lambda}-\sqrt{\mu}}{\sqrt{\lambda}+\sqrt{\mu}}\left(z^{j+1}-z^{j}\right),
\end{aligned}
$$

where $0<\mu I \leq H \leq \lambda I$.

\section{B. $A D M M$}

One of the most popular methods for problem (1) is ADMM [9], [19]-[21], which iterates as follows

$$
\begin{aligned}
y^{k+1} & =\underset{z: G z=g}{\operatorname{argmin}} \frac{1}{2} z^{\top} H z+h^{\top} z+\frac{1}{2 \alpha}\left\|z+w^{k}-y^{k}\right\|_{2}^{2}, \\
z^{k+1} & =\pi_{\mathbb{Z}}\left[y^{k+1}+w^{k}\right] \\
w^{k} & =w^{k}+z^{k+1}-y^{k+1} .
\end{aligned}
$$

Notice that ADMM solves two subproblems for primal variables: minimization of a quadratic function over a hyperplane in (4a) and the projection in (4b). The minimization in 4a is equivalent to solving the following system of linear equations for variable $z$

$$
\left[\begin{array}{cc}
H+\frac{1}{\alpha} I & G^{\top} \\
G & 0
\end{array}\right]\left[\begin{array}{l}
z \\
v
\end{array}\right]=\left[\begin{array}{c}
-h-\frac{1}{\alpha}\left(w^{k}-y^{k}\right) \\
g
\end{array}\right],
$$

which requires pre-computing either matrix inverse [9] or LDL decomposition [19]. If both matrix $H$ and $G$ are time invariant, such pre-computation only needs to be executed once. However, for time varying applications, e.g., those from nonlinear MPC [29], such precomputation needs to be executed every time matrix $H$ or $G$ is updated.

\section{Chambolle \& Pock method}

The Chambolle \& Pock method [18] has been used to solve problem (1) through the following iterates

$$
z^{k+1}=\underset{z \in \mathbb{Z}}{\operatorname{argmin}} \frac{1}{2} z^{\top} H z+h^{\top} z+\frac{1}{2 \alpha}\left\|z+\alpha G^{\top} w^{k}-z^{k}\right\|_{2}^{2}
$$

$w^{k+1}=w^{k}+\alpha\left(G\left(2 z^{k+1}-z^{k}\right)-g\right)$.

Recently, a more efficient variant of (6) was introduced in [26], which replaces 6a with a single projection onto $\mathbb{Z}$. If $H \geq 0$ in (1), this method achieves $O(1 / k)$ convergence rate using the following iterates [26, Alg. 1]

$$
\begin{aligned}
z^{k+1} & =\pi_{\mathbb{Z}}\left[z^{k}-\alpha\left(H z^{k}+h+G^{\top} w^{k}\right)\right] \\
w^{k+1} & =w^{k}+\beta\left(G\left(2 z^{k+1}-z^{k}\right)-g\right) .
\end{aligned}
$$

Furthermore, if $H>0$, an improved convergence rate of $O\left(1 / k^{2}\right)$ is possible using the following iterates [26, Alg. 4]

$$
\begin{aligned}
w^{k+1} & =w^{k}+\beta^{k}\left(G\left(z^{k}+\gamma^{k}\left(z^{k}-z^{k-1}\right)\right)-g\right), \\
z^{k+1} & =\pi_{\mathbb{Z}}\left[z^{k}-\frac{\alpha^{k}}{\mu \alpha^{k}+1}\left(H z^{k}+h+G^{\top} w^{k+1}\right)\right]
\end{aligned}
$$

where step sizes $\left(\alpha^{k}, \beta^{k}, \gamma^{k}\right)$ are computed recursively; see [26, Sec. 5.2] for details. A different version of (7) was introduced in [25], where (7b) is replaced with $w^{k+1}=w^{k}+$ $\beta\left(G z^{k+1}-g\right)$. However, no convergence rate was provided in [25]. On the other hand, the convergence of algorithm (7) and (8) are both proved using a non-negative running duality gap function [26, Thm. 1, Thm. 4]. However, these results provide no convergence rates on constraint violation amount, namely $\left\|G z^{k}-g\right\|_{2}^{2}$. 


\section{Proportional-integral PRojected GRAdient METHOD}

We now introduce proportional-integral projected gradient method for problem (1). We will show that, along certain sequences of averaged iterates, our method achieves a convergence rate of $O(1 / k)$ and $O\left(1 / k^{2}\right)$ when matrix $H$ is positive semi-definite and, respectively, positive definite.

The method we propose iterates as follows

$$
\begin{aligned}
v^{k} & =w^{k}+\beta^{k}\left(G z^{k}-g\right), \\
z^{k+1} & =\pi_{\mathbb{Z}}\left[z^{k}-\alpha^{k}\left(H z^{k}+h+G^{\top} v^{k}\right)\right], \\
w^{k+1} & =w^{k}+\beta^{k}\left(G z^{k+1}-g\right) .
\end{aligned}
$$

Remark 1. Notice that (9a) and (9c) compute a proportional and, respectively, integral feedback of the affine constraints violation. Hence an intuitive interpretation of (9) is applying projected gradient method to variable $z$, where the gradient is corrected by a proportional-integral (PI) feedback. Similar PI feedback was also used in distributed optimization algorithms [27], [30]-[32].

We will prove that method (9) achieves $O(1 / k)$ and $O\left(1 / k^{2}\right)$ convergence rate when matrix $H$ is positive semidefinite and, respectively, positive definite. The latter is optimal for solving problem (1) using first order methods [33, Thm.1.1].

We now prove the convergence properties of method 9 . First, we group our assumptions as follows.

\section{Assumption 1. Suppose}

1) set $\mathbb{Z} \subset \mathbb{R}^{n}$ is closed and convex; matrix $H \in \mathbb{R}^{n \times n}$ is symmetric, matrix $G \in \mathbb{R}^{m \times n}$ has full row rank; there exists $\mu, \lambda, \sigma \in \mathbb{R}$ with $0 \leqslant \mu \leqslant \lambda$ and $\sigma \geqslant 0$ such that $\mu I \leq H \leq \lambda I$ and $G^{\top} G \leq \sigma I$.

2) there exists $z^{\star} \in \mathbb{R}^{n}$ and $w^{\star} \in \mathbb{R}^{m}$ such that

$$
\begin{gathered}
G z^{\star}=g, \quad z^{\star} \in \mathbb{Z}, \\
\left\langle H z^{\star}+h+G^{\top} w^{\star}, z-z^{\star}\right\rangle \geqslant 0, \quad \forall z \in \mathbb{Z} .
\end{gathered}
$$

Remark 2. Equation (10) gives the Karush-Kuhn-Tucker conditions of problem (1). Under the Slater condition for equalities, equation (10) holds if and only if $z^{\star}$ is an optimal solution for problem (11); see [28, Thm.3.1.27].

We will use the following result on Euclidean projection.

Lemma 1. [28, Lemma. 2.2.7] If set $\mathbb{Z} \subset \mathbb{R}^{n}$ is closed and convex, then

$$
\left\langle\pi_{\mathbb{Z}}[z]-z, z^{\prime}-\pi_{\mathbb{Z}}[z]\right\rangle \geqslant 0, \quad \forall z \in \mathbb{R}^{n}, z^{\prime} \in \mathbb{Z} .
$$

The following lemma shows the key property of any two consecutive iterations generated by method (9).

Lemma 2. Suppose Assumption 1 holds and sequence $\left\{z^{k}, w^{k}\right\}$ is generated by (9). If $\lambda+\sigma \beta^{k}=\frac{1}{\alpha^{k}}$ for all $k \geqslant 1$, then

$$
\begin{aligned}
& \quad \frac{\beta^{k}}{2}\left\|G z^{k}-g\right\|_{2}^{2}+\frac{1}{2}\left\|z^{k+1}-z^{\star}\right\|_{H}^{2} \\
& \leqslant \frac{1}{2}\left(\frac{1}{\alpha^{k}}-\mu\right)\left\|z^{k}-z^{\star}\right\|_{2}^{2}+\frac{1}{2 \beta^{k}}\left\|w^{k}-w^{\star}\right\|_{2}^{2} \\
& \quad-\frac{1}{2 \alpha^{k}}\left\|z^{k+1}-z^{\star}\right\|_{2}^{2}-\frac{1}{2 \beta^{k}}\left\|w^{k+1}-w^{\star}\right\|_{2}^{2} .
\end{aligned}
$$

Proof. First, applying Lemma 1 to $9 \mathrm{~b}$ gives

$$
\begin{aligned}
0 \leqslant & \frac{1}{\alpha^{k}}\left\langle z^{k+1}-z^{k}, z^{\star}-z^{k+1}\right\rangle+\beta^{k}\left\langle G z^{k}-g, g-G z^{k+1}\right\rangle \\
& +\left\langle H z^{k}+h, z^{\star}-z^{k+1}\right\rangle+\left\langle w^{k}, G\left(z^{\star}-z^{k+1}\right)\right\rangle,
\end{aligned}
$$

where we also used (9a) and (10a). Next, (10b) implies that

$$
0 \leqslant-\left\langle H z^{\star}+h, z^{\star}-z^{k+1}\right\rangle-\left\langle w^{\star}, G\left(z^{\star}-z^{k+1}\right)\right\rangle .
$$

In addition, one can directly verify the following four identities, which can be interpreted as instances of the law of cosines; see Fig. 1 for an illustration.

$$
\begin{aligned}
&\left\langle z^{k+1}-z^{k}, z^{\star}-z^{k+1}\right\rangle \\
&= \frac{1}{2}\left\|z^{k}-z^{\star}\right\|_{2}^{2}-\frac{1}{2}\left\|z^{k+1}-z^{\star}\right\|_{2}^{2}-\frac{1}{2}\left\|z^{k+1}-z^{k}\right\|_{2}^{2}, \\
&\left\langle G z^{k}-g, g-G z^{k+1}\right\rangle \\
&=\frac{1}{2}\left\|G\left(z^{k+1}-z^{k}\right)\right\|_{2}^{2}-\frac{1}{2}\left\|G z^{k}-g\right\|_{2}^{2}-\frac{1}{2}\left\|G z^{k+1}-g\right\|_{2}^{2}, \\
&\left\langle H^{\frac{1}{2}}\left(z^{k}-z^{\star}\right), H^{\frac{1}{2}}\left(z^{\star}-z^{k+1}\right)\right\rangle \\
&= \frac{1}{2}\left\|z^{k+1}-z^{k}\right\|_{H}^{2}-\frac{1}{2}\left\|z^{k}-z^{\star}\right\|_{H}^{2}-\frac{1}{2}\left\|z^{k+1}-z^{\star}\right\|_{H}^{2},(15) \\
& \frac{1}{2}\left\|w^{k+1}-w^{\star}\right\|_{2}^{2}-\frac{1}{2}\left\|w^{k}-w^{\star}\right\|_{2}^{2} \\
&=\left\langle w^{k}-w^{\star}, w^{k+1}-w^{k}\right\rangle+\frac{1}{2}\left\|w^{k+1}-w^{k}\right\|_{2}^{2} \\
&= \beta^{k}\left\langle w^{k}-w^{\star}, G\left(z^{k+1}-z^{\star}\right)\right\rangle+\frac{\left(\beta^{k}\right)^{2}}{2}\left\|G z^{k+1}-g\right\|_{2}^{2},
\end{aligned}
$$

where matrix $H^{\frac{1}{2}}$ in $(15)$ is the positive semi-definite square root of $H$, and the last step in 16 is due to $9 \mathrm{c}$ and $10 \mathrm{a}$. Further, the assumption that $0 \leq \mu I \leq H \leq \lambda I$ and $G^{\top} G \leq$

$$
\underset{c}{b} a \quad-a b \cos \theta=\frac{1}{2} c^{2}-\frac{1}{2} a^{2}-\frac{1}{2} b^{2}
$$

Fig. 1: The law of cosines.

$\sigma I$ implies the following

$$
\begin{aligned}
\frac{\mu}{2}\left\|z^{k}-z^{\star}\right\|_{2}^{2} & \leqslant \frac{1}{2}\left\|z^{k}-z^{\star}\right\|_{H}^{2}, \\
\frac{1}{2}\left\|z^{k+1}-z^{k}\right\|_{H}^{2} & \leqslant \frac{\lambda}{2}\left\|z^{k+1}-z^{k}\right\|_{2}^{2}, \\
\frac{1}{2}\left\|G\left(z^{k+1}-z^{k}\right)\right\|_{2}^{2} & \leqslant \frac{\sigma}{2}\left\|z^{k+1}-z^{k}\right\|_{2}^{2} .
\end{aligned}
$$

Finally, summing up together (11), (12), $\frac{1}{\alpha^{k}} \times(13), \beta^{k} \times(14)$, $\left.(15), \frac{1}{\beta^{k}} \times(16),(17 \mathrm{a}), \sqrt{17 \mathrm{~b}}\right)$ and $\beta^{k} \times(17 \mathrm{c})$, then using the assumption that $\lambda+\sigma \beta^{k}=\frac{1}{\alpha^{k}}$, we obtain the desired result.

With the above lemma, we are now ready to prove the convergence properties of method $(9)$ under different assumptions on matrix $H$. We start with the case where matrix $H$ is only positive semi-definite, i.e., $\mu=0$ in Assumption 1 The following theorem shows that, along a sequence of averaged iterates generated by (9), both the quadratic distance to optimum and constraint violation converge to zero at the rate of $O(1 / k)$.

Theorem 1. Suppose Assumption 1 hold with $\mu=0$, and sequence $\left\{v^{k}, z^{k}, w^{k}\right\}$ is generated by (9) with $\alpha^{k}=\frac{1}{\beta \sigma+\lambda}$ 
and $\beta^{k}=\beta$ for some $\beta>0$ and all $k \geqslant 1$. Let $V^{1}=$ $\frac{1}{2 \alpha}\left\|z^{1}-z^{\star}\right\|_{2}^{2}+\frac{1}{2 \beta}\left\|w^{1}-w^{\star}\right\|_{2}^{2}$, then

$$
\frac{1}{2}\left\|G \hat{z}^{k}-g\right\|_{2}^{2} \leqslant \frac{1}{\beta k} V^{1}, \quad \frac{1}{2}\left\|\tilde{z}^{k}-z^{\star}\right\|_{H}^{2} \leqslant \frac{1}{k} V^{1} .
$$

where $\hat{z}^{k}=\frac{1}{k} \sum_{j=1}^{k} z^{j}$ and $\tilde{z}^{k}=\frac{1}{k} \sum_{j=1}^{k} z^{j+1}$.

Proof. With this choice of $\alpha^{k}$ and $\beta^{k}$, the inequality in Lemma 2 becomes the following: for all $j \geqslant 1$,

$$
\frac{\beta}{2}\left\|G z^{j}-g\right\|_{2}^{2}+\frac{1}{2}\left\|z^{j+1}-z^{\star}\right\|_{H}^{2} \leqslant V^{j}-V^{j+1},
$$

where $V^{j}=\frac{1}{2 \alpha}\left\|z^{j}-z^{\star}\right\|_{2}^{2}+\frac{1}{2 \beta}\left\|w^{j}-w^{\star}\right\|_{2}^{2}$. Summing up this inequality for $j=1, \ldots, k$ gives

$$
\begin{aligned}
& \sum_{j=1}^{k}\left(\frac{\beta}{2}\left\|G z^{j}-g\right\|_{2}^{2}+\frac{1}{2}\left\|z^{j+1}-z^{\star}\right\|_{H}^{2}\right) \\
\leqslant & V^{1}-V^{k+1} \leqslant V^{1}
\end{aligned}
$$

where the last step is because $V^{k+1} \geqslant 0$. Hence

$$
\frac{\beta}{2} \sum_{j=1}^{k}\left\|G z^{j}-g\right\|_{2}^{2} \leqslant V^{1}, \quad \frac{1}{2} \sum_{j=1}^{k}\left\|z^{j+1}-z^{\star}\right\|_{H}^{2} \leqslant V^{1},
$$

Finally, applying Jensen's inequality to the above two inequalities gives the desired results.

If matrix $H$ is positive definite, i.e., $\mu>0$ in Assumption 1 . then, along two different sequences of averaged iterates, the $O(1 / k)$ rate in Theorem 1 can be improved to $O\left(1 / k^{2}\right)$ for the quadratic distance to optimum and $O\left(1 / k^{3}\right)$ for constraint violation.

Theorem 2. Suppose Assumption 1 hold with $\mu>0$, and sequence $\left\{v^{k}, z^{k}, w^{k}\right\}$ is generated by [9] with $\alpha^{k}=\frac{2}{(k+1) \mu+2 \lambda}$, $\beta^{k}=\frac{(k+1) \mu}{2 \sigma}$ for all $k \geqslant 1$. Let $V^{1}=\frac{1}{2(\mu+\lambda)}\left\|z^{1}-z^{\star}\right\|_{2}^{2}+$ $\frac{\sigma}{2 \mu}\left\|w^{1}-w^{\star}\right\|_{2}^{2}$, then

$$
\begin{aligned}
& \frac{1}{2}\left\|G \hat{z}^{k}-g\right\|_{2}^{2} \leqslant \frac{12 \lambda \sigma}{\mu^{2} k\left(k^{2}+6 k+11\right)} V^{1} \\
& \frac{1}{2}\left\|\tilde{z}^{k}-z^{\star}\right\|_{H}^{2} \leqslant \frac{4 \lambda}{\mu k(k+5)} V^{1}
\end{aligned}
$$

where $\hat{z}^{k}=\frac{3}{k\left(k^{2}+6 k+11\right)} \sum_{j=1}^{k}(j+1)(j+2) z^{j}$ and $\tilde{z}^{k}=$ $\frac{2}{k(k+5)} \sum_{j=1}^{k}(j+2) z^{j+1}$.

Proof. With this choice of $\alpha^{k}$ and $\beta^{k}$, the inequality in Lemma 2 becomes the following: for all $j \geqslant 1$,

$$
\begin{aligned}
& \frac{(j+1) \mu}{4 \sigma}\left\|G z^{j}-g\right\|_{2}^{2}+\frac{1}{2}\left\|z^{j+1}-z^{\star}\right\|_{H}^{2} \\
\leqslant & \frac{1}{2}\left(\frac{1}{\alpha^{j}}-\mu\right)\left\|z^{j}-z^{\star}\right\|_{2}^{2}+\frac{1}{2 \beta^{j}}\left\|w^{j}-w^{\star}\right\|_{2}^{2}-V^{j+1},
\end{aligned}
$$

where $V^{j}=\frac{1}{2 \alpha^{j-1}}\left\|z^{j}-z^{\star}\right\|_{2}^{2}+\frac{1}{2 \beta^{j-1}}\left\|w^{j}-w^{\star}\right\|_{2}^{2}$. Let $\kappa=$ $\lambda / \mu \geqslant 1$, then it is straightforward to verify the following

$$
\begin{aligned}
\left(\frac{1}{\alpha^{j}}-\mu\right)(j+2 \kappa) & =\frac{1}{\alpha^{j-1}}(j+2 \kappa-1), \\
\frac{1}{\beta^{j}}(j+2 \kappa) & \leqslant \frac{1}{\beta^{j-1}}(j+2 \kappa-1) .
\end{aligned}
$$

Hence multiplying (18) with $(j+2 \kappa)$ and substituting in (19) we can show

$$
\begin{aligned}
& \frac{(j+1)(j+2 \kappa) \mu}{4 \sigma}\left\|G z^{j}-g\right\|_{2}^{2}+\frac{j+2 \kappa}{2}\left\|z^{j+1}-z^{\star}\right\|_{H}^{2} \\
\leqslant & (j+2 \kappa-1) V^{j}-(j+2 \kappa) V^{j+1} .
\end{aligned}
$$

Summing up this inequality for $j=1,2, \ldots, k$ gives

$$
\begin{aligned}
& \sum_{j=1}^{k}\left(\frac{(j+1)(j+2 \kappa) \mu}{4 \sigma}\left\|G z^{j}-g\right\|_{2}^{2}+\frac{j+2 \kappa}{2}\left\|z^{j+1}-z^{\star}\right\|_{H}^{2}\right) \\
\leqslant & 2 \kappa V^{1}-(k+2 \kappa) V^{k+1} \leqslant 2 \kappa V^{1} .
\end{aligned}
$$

where the last step is because $V^{k+1} \geqslant 0$. Since $\kappa \geqslant 1$, the above inequality implies the following

$$
\begin{aligned}
\sum_{j=1}^{k} \frac{(j+1)(j+2) \mu}{4 \sigma}\left\|G z^{j}-g\right\|_{2}^{2} & \leqslant 2 \kappa V^{1}, \\
\sum_{j=1}^{k} \frac{j+2}{2}\left\|z^{j+1}-z^{\star}\right\|_{H}^{2} & \leqslant 2 \kappa V^{1} .
\end{aligned}
$$

Finally, applying Jensen's inequality to the above two inequalities and using $\kappa=\lambda / \mu$ gives the desired results.

Notice that Theorem 2, in contrast to Theorem 11, uses a weighted average of iterates similar to those in subgradient method [34] and accelerated ADMM [35].

Remark 3. Theorem 2 not only establishes the $O\left(1 / k^{2}\right)$ convergence rate of the quadratic distance to the optimum, but also shows the $O\left(1 / k^{3}\right)$ convergence rate of the constraint violation. To our best knowledge, the latter has never been proven for any existing methods that achieve $O\left(1 / k^{2}\right)$ convergence rate [8], [16], [17], [25]-[27].

\section{EFFICIENT IMPLEMENTATION FOR MPC}

In this section, we provide the pseudocode implementation of method (9) for the following tracking problem

$$
\begin{array}{ll}
\underset{\left\{u_{t-1}, x_{t}\right\}_{t=1}^{T}}{\operatorname{minimize}} & \frac{1}{2} \sum_{t=1}^{T}\left\|x_{t}-y_{t}\right\|_{Q_{t}}^{2}+\frac{1}{2} \sum_{t=0}^{T-1}\left\|u_{t}\right\|_{R_{t}}^{2} \\
\text { subject to } & x_{t}=A_{t-1} x_{t-1}+B_{t-1} u_{t-1}, \\
& u_{t-1} \in \mathbb{U}_{t-1}, x_{t} \in \mathbb{X}_{t}, \quad 1 \leqslant t \leqslant T .
\end{array}
$$

where, for all $1 \leqslant t \leqslant T$ : closed convex sets $\mathbb{X}_{t} \subset \mathbb{R}^{n_{x}}$ and $\mathbb{U}_{t-1} \subset \mathbb{R}^{n_{u}}$ describe feasible sets for state variable $x_{t}$ and, respectively, input variable $u_{t-1} ; A_{t-1} \in \mathbb{R}^{n_{x} \times n_{x}}, B_{t-1} \in$ $\mathbb{R}^{n_{x} \times n_{u}}$ describe the linear dynamics of the plant; $y_{t}$ gives the reference value for $x_{t}$.

We first rewrite problem 20 as a special case of problem (1) by defining the following

$$
\begin{aligned}
z & =\left[u_{0}^{\top}, x_{1}^{\top}, \ldots, u_{T-1}^{\top}, x_{T}^{\top}\right]^{\top}, \mathbb{Z}=\prod_{t=1}^{T}\left(\mathbb{U}_{t-1} \times \mathbb{X}_{t}\right), \\
H & =\operatorname{blkdiag}\left(R_{0}, Q_{1}, \ldots, R_{T-1}, Q_{T}\right), \\
h & =\left[0^{\top},-y_{1}^{\top} Q_{1}, \ldots, 0^{\top},-y_{T}^{\top} Q_{T}\right]^{\top}, \\
G & =\left[\begin{array}{ccc}
-B_{0} & \\
-A_{1}-B_{1} & I & \\
\ddots & \ddots & \ddots \\
-A_{T-1}-B_{T-1} I
\end{array}\right] \\
g & =\left[x_{0}^{\top} A_{0}^{\top}, 0^{\top}, \cdots, 0^{\top}\right]^{\top} .
\end{aligned}
$$

We are now ready to implement 9 for problem 20. We partition variables $w$ and $v$ as follows

$$
v=\left[v_{1}^{\top}, v_{2}, \ldots, v_{T}^{\top}\right]^{\top}, w=\left[w_{1}^{\top}, w_{2}, \ldots, w_{T}^{\top}\right]^{\top},
$$

where $v_{t}, w_{t} \in \mathbb{R}^{n_{x}}$ corresponds to constraint $x_{t}=$ $A_{t-1} x_{t-1}+B_{t-1} u_{t-1}$ for $1 \leqslant t \leqslant T$. In addition, the separable structure of set $\mathbb{Z}$ defined by 21 allows separable computation of its Euclidean projection. Based on these observations, we implement algorithm 9. for problem 20. in Algorithm 1, where we introduce dummy parameters $A_{T} v_{T+1} \equiv 0$ to simplify our notation. Notice that updates of variables corresponding to different value of $t$ can be executed in parallel, hence the algorithm run-time can be almost independent of horizon $T$. 


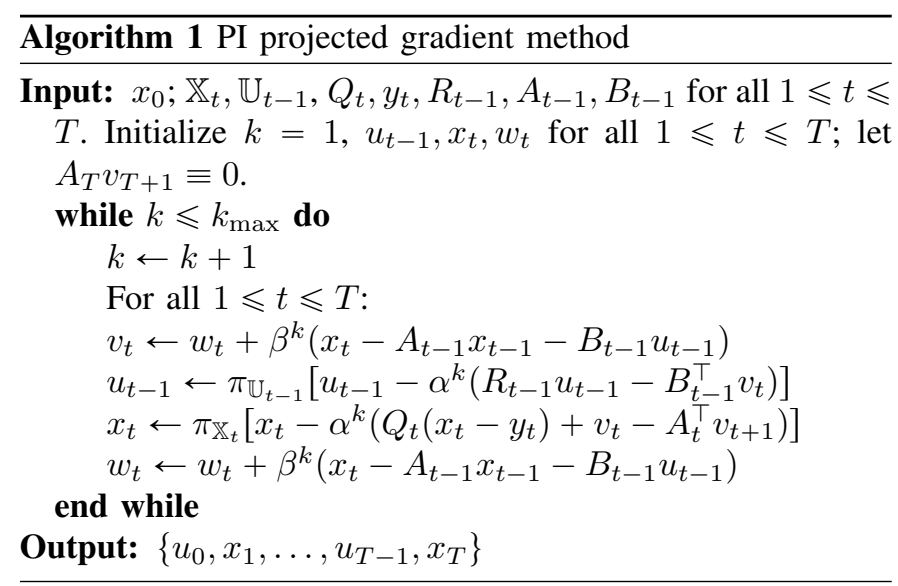

\section{NUMERICAL EXAMPLES}

In this section we compare our method against the existing methods reviewed in Section III over a trajectory-planning problem with keep-out-zone constraints, where all parameters are chosen as unit-less for simplicity.

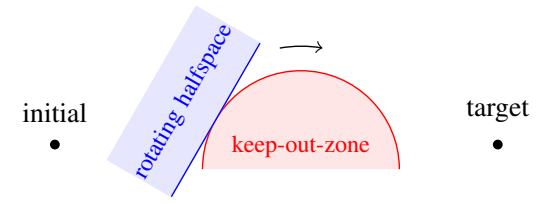

Fig. 2: Trajectory-planning with rotating halfspace constraint.

We consider a trajectory-planning (finite horizon optimal control) problem where the goal is to track a beeline trajectory from initial to target position while avoiding collision with a circular keep-out-zone; see Fig. 2 for an illustration. Here, this problem is an instant of an MPC problem, which is solved repetitively as new state information becomes available. The dynamics of the system is modeled as a double integrator with sampling time $0.5 \mathrm{~s}$. The system is subject to $\ell_{2}$ norm constraints on its velocity $q \in \mathbb{R}^{2}$ and acceleration input $u \in \mathbb{R}^{2}$. In addition, a rotating half-space constraint is imposed on its position $p \in \mathbb{R}^{2}$, which convexifies the keep-out-zone; see [36] for a detailed discussion. We model this tracking problem as a special case of problem 20] with the following choice of parameters:

$$
\begin{aligned}
& A_{t-1}=\left[\begin{array}{cccc}
1 & 0 & 0.5 & 0 \\
0 & 1 & 0 & 0.5 \\
0 & 0 & 1 & 0 \\
0 & 0 & 0 & 1
\end{array}\right], B_{t-1}=\left[\begin{array}{cc}
0.125 & 0 \\
0 & 0.125 \\
0.5 & 0 \\
0 & 0.5
\end{array}\right], \\
& Q_{t}=\operatorname{diag}(1,0.5,1,0.5), R_{t-1}=\operatorname{diag}(1,0.5), \\
& \mathbb{X}_{t}=\left\{x=\left[\begin{array}{l}
p \\
q
\end{array}\right] \mid\left[\begin{array}{c}
-\cos (\theta t) \\
\sin (\theta t)
\end{array}\right]^{\top} p \geqslant 2,\|q\|_{2} \leqslant 0.25\right\}, \\
& \mathbb{U}_{t-1}=\left\{u \mid\|u\|_{2} \leqslant 0.1\right\}, \quad x_{0}=\left[\begin{array}{llll}
-2.5 & 0.6 & 0 & 0
\end{array}\right]^{\top},
\end{aligned}
$$

for $1 \leqslant t \leqslant T$, where $\theta=0.063$ in $(23 \mathrm{c}$ ) is a constant rotation rate [36]. Note that $Q_{t}$ and $R_{t}$ in (23b) are diagonal but not identity, which is common in practice. The reference trajectory $\left\{y_{t}\right\}_{t=1}^{T}$ in 200 is chosen as a beeline trajectory from initial position $(-2.5,0.6)$ to target position $(2.9,0.3)$ without considering the position constraint on $p$ in 23c.
We compare our method against all the other methods reviewed in Section II In terms of step sizes: for our method (9), we choose $\alpha^{k}$ and $\beta^{k}$ according to Theorem 1 and Theorem 2 for constant and, respectively, varying step sizes; for dual fast gradient method, we choose $\alpha$ according to [8, Thm.1]; for Chambolle \& Pock method (C \& P), we choose the constant step sizes in (7) according to [26, Rem. 1], and the varying step sizes in (8) according to the "optimal rule" in [26, Sec. 5.2]; for ADMM, we choose $\alpha=2$ as suggested in [9]. In addition, the inner loop iterations used by each iteration of method (2) are warm-started using results from the last outer iteration and terminated if $\left\|z^{j+1}-z^{j}\right\|_{2} /\left\|z^{j}\right\|_{2} \leqslant \epsilon_{\text {inner }}$, where $\epsilon_{\text {inner }}$ is chosen between $0.1 \%$ and $0.01 \%$.

We summarize our results as follows. Fig. 3 shows the convergence over iterations of different algorithms with same initialization for $T=25$, where $z^{\star}$ is computed using ECOS [37] together with JuMP [38]. Fig. 4 shows the computation costed by different algorithms for $T=\{5,15,25,35,45\}$ to reach the tolerance for constraint violation (we use $\ell_{\infty}$-norm since it measures the maximum pointwise constraint violation along the trajectory), where each data point is averaged over 200 independent experiments using initialization sampled from standard normal distribution. Note that we omitted method (7) and (9) with constant step sizes in Fig. $4 \mathrm{~b}$ due to their slow convergence.

In these simulations, our method with varying step sizes (var.) outperforms the others. Our method with constant step sizes (const.) converges slower, but is almost identically to Chambolle \& Pock method (C \& P) with constant step sizes (const.), since they do not exploit the strong convexity of the objective functions.

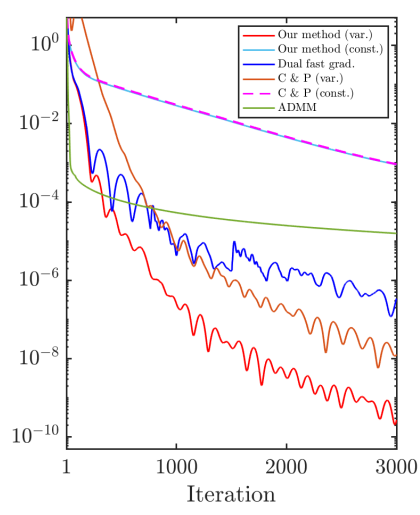

(a) $\left\|z-z^{\star}\right\|_{2}^{2}$.

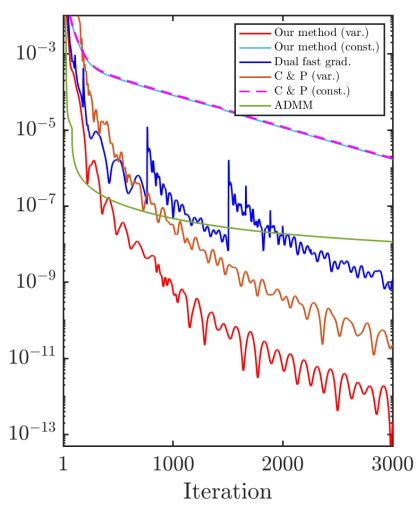

(b) $\|G z-g\|_{2}^{2}$.
Fig. 3: Convergence over iterations for $T=25$.

\section{CONCLUSION}

We introduced a novel first order primal-dual method for MPC, which uses a single projection onto the state and input constraint set per-iteration. We prove the convergence rate of both the distance to optimum and the constraint violation along different sequences of averaged iterates. Our method not only enjoys simple interpretation based on PI feedback, but also outperforms existing methods in numerical experiments. 


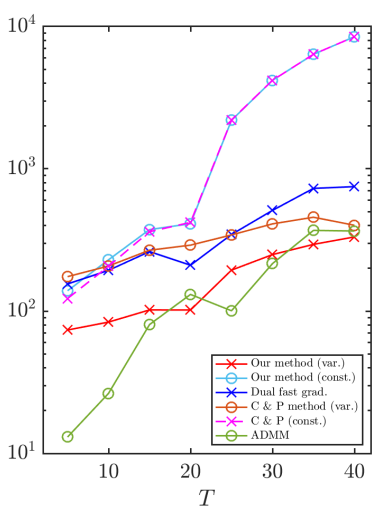

(a) $\epsilon=10^{-3}$.

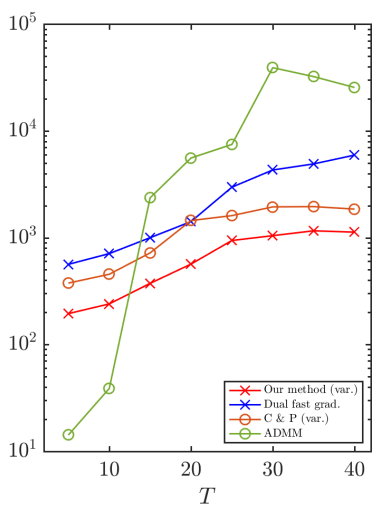

(b) $\epsilon=10^{-5}$.
Fig. 4: Number of projection $\pi_{\mathbb{Z}}[\cdot]$ costed to reach condition $\|G z-g\|_{\infty} \leqslant \epsilon$. Each data point is averaged over 200 simulations using random initialization.

Future directions include real-time implementation, faster empirical convergence using preconditioning, and other controlinspired optimization algorithms.

\section{REFERENCES}

[1] D. Q. Mayne, J. B. Rawlings, C. V. Rao, and P. O. Scokaert, "Constrained model predictive control: Stability and optimality," Automatica, vol. 36, no. 6, pp. 789-814, 2000.

[2] D. Q. Mayne, "Model predictive control: Recent developments and future promise," Automatica, vol. 50, no. 12, pp. 2967-2986, 2014.

[3] S. J. Qin and T. A. Badgwell, "A survey of industrial model predictive control technology," Control. Eng. Pract., vol. 11, no. 7, pp. 733-764, 2003.

[4] S. Kouro, P. Cortés, R. Vargas, U. Ammann, and J. Rodríguez, "Model predictive control-a simple and powerful method to control power converters," IEEE Trans. Ind. Electron., vol. 56, no. 6, pp. 1826-1838, 2008.

[5] U. Eren, A. Prach, B. B. Koçer, S. V. Raković, E. Kayacan, and B. Açıkmeşe, "Model predictive control in aerospace systems: Current state and opportunities," J. Guid. Control Dyn., vol. 40, no. 7, pp. 15411566, 2017.

[6] F. Ullmann, "FiOrdOs: A Matlab toolbox for C-code generation for first order methods," Master's thesis, Dept. Inf. Technol. Elect. Eng., ETH Zurich, 2011.

[7] S. Richter, C. N. Jones, and M. Morari, "Computational complexity certification for real-time MPC with input constraints based on the fast gradient method," IEEE Trans. Automat. Control, vol. 57, no. 6, pp. 1391-1403, 2011.

[8] — " "Certification aspects of the fast gradient method for solving the dual of parametric convex programs," Math. Meth. Oper. Res., vol. 77, no. 3, pp. 305-321, 2013.

[9] J. L. Jerez, P. J. Goulart, S. Richter, G. A. Constantinides, E. C. Kerrigan, and M. Morari, "Embedded online optimization for model predictive control at megahertz rates," IEEE Trans. Automat. Control, vol. 59, no. 12 , pp. $3238-3251,2014$.

[10] H. H. Bauschke, "Projection algorithms and monotone operators," Ph.D. dissertation, Dept. Math. and Statist., Simon Fraser Univ., 1996.

[11] R. Ghaemi, J. Sun, and I. V. Kolmanovsky, "An integrated perturbation analysis and sequential quadratic programming approach for model predictive control," Automatica, vol. 45, no. 10, pp. 2412-2418, 2009.

[12] R. Bonalli, A. Cauligi, A. Bylard, and M. Pavone, "Gusto: Guaranteed sequential trajectory optimization via sequential convex programming," in Int. Conf. Robot. Autom. IEEE, 2019, pp. 6741-6747.

[13] Y. Mao, M. Szmuk, and B. Açıkmeşe, "Successive convexification of non-convex optimal control problems and its convergence properties," in IEEE Conf. Decision Control. IEEE, 2016, pp. 3636-3641.

[14] Y. Mao, D. Dueri, M. Szmuk, and B. Açıkmeșe, "Successive convexification of non-convex optimal control problems with state constraints," IFAC-PapersOnLine, vol. 50, no. 1, pp. 4063-4069, 2017.
[15] Y. Mao, M. Szmuk, X. Xu, and B. Açikmese, "Successive convexification: A superlinearly convergent algorithm for non-convex optimal control problems," arXiv preprint arXiv:1804.06539[math.OC], 2018.

[16] P. Patrinos and A. Bemporad, "An accelerated dual gradient-projection algorithm for embedded linear model predictive control," IEEE Trans. Automat. Control, vol. 59, no. 1, pp. 18-33, 2013.

[17] P. Giselsson, "Improved fast dual gradient methods for embedded model predictive control," IFAC Proc. Vol., vol. 47, no. 3, pp. 2303-2309, 2014.

[18] D. K. M. Kufoalor, S. Richter, L. Imsland, T. A. Johansen, M. Morari, and G. O. Eikrem, "Embedded model predictive control on a PLC using a primal-dual first-order method for a subsea separation process," in Proc. Mediterranean Conf. Control Autom. IEEE, 2014, pp. 368-373.

[19] B. O'Donoghue, G. Stathopoulos, and S. Boyd, "A splitting method for optimal control," IEEE Trans. Control Syst. Technol., vol. 21, no. 6, pp. 2432-2442, 2013.

[20] L. E. Sokoler, G. Frison, M. S. Andersen, and J. B. Jørgensen, "Inputconstrained model predictive control via the alternating direction method of multipliers," in Proc. Eur. Control Conf. IEEE, 2014, pp. 115-120.

[21] T. V. Dang, K. V. Ling, and J. M. Maciejowski, "Embedded ADMMbased QP solver for MPC with polytopic constraints," in Proc. Eur. Control Conf. IEEE, 2015, pp. 3446-3451.

[22] Y. Wang and S. Boyd, "Fast model predictive control using online optimization," IEEE Trans. Control Syst. Technol., vol. 18, no. 2, pp. 267-278, 2009.

[23] M. Kögel and R. Findeisen, "Fast predictive control of linear systems combining Nesterov's gradient method and the method of multipliers," in Proc. IEEE Conf. Decision Control and Eur. Control Conf. IEEE, 2011, pp. 501-506.

[24] P. Patrinos, P. Sopasakis, and H. Sarimveis, "A global piecewise smooth Newton method for fast large-scale model predictive control," Automatica, vol. 47, no. 9, pp. 2016-2022, 2011.

[25] H. A. Blanchard and A. A. Adegbege, "An SOR-like method for fast model predictive control," IFAC-PapersOnLine, vol. 50, no. 1, pp. 14 418-14 423, 2017.

[26] A. Chambolle and T. Pock, "On the ergodic convergence rates of a first-order primal-dual algorithm," Math. Program., vol. 159, no. 1-2, pp. 253-287, 2016.

[27] J. H. Seidman, M. Fazlyab, G. J. Pappas, and V. M. Preciado, "A chebyshev-accelerated primal-dual method for distributed optimization," in Proc. IEEE Conf. Decision Control. IEEE, 2018, pp. 1775-1781.

[28] Y. Nesterov, Lectures on convex optimization. Springer, 2010, vol. 137.

[29] D. Kouzoupis, G. Frison, A. Zanelli, and M. Diehl, "Recent advances in quadratic programming algorithms for nonlinear model predictive control," Vietnam J. Math., vol. 46, no. 4, pp. 863-882, 2018.

[30] J. Wang and N. Elia, "Control approach to distributed optimization," in Proc. Allerton Conf. Commun. Control Comput. IEEE, 2010, pp. 557-561.

[31] Y. Yu, B. Açıkmeşe, and M. Mesbahi, "Mass-spring-damper networks for distributed optimization in non-Euclidean spaces," Automatica, vol. 112, p. 108703, 2020.

[32] Y. Yu and B. Açıkmeşe, "RLC circuits-based distributed mirror descent method," IEEE Control Syst. Lett., vol. 4, no. 3, pp. 548-553, 2020.

[33] Y. Ouyang and Y. Xu, "Lower complexity bounds of first-order methods for convex-concave bilinear saddle-point problems," Math. Program., pp. $1-35,2019$.

[34] S. Lacoste-Julien, M. Schmidt, and F. Bach, "A simpler approach to obtaining an $\mathrm{O}(1 / \mathrm{t})$ convergence rate for the projected stochastic subgradient method," arXiv preprint arXiv:1212.2002[cs.LG], 2012.

[35] Y. Xu, "Accelerated first-order primal-dual proximal methods for linearly constrained composite convex programming," SIAM J. Optim., vol. 27, no. 3, pp. 1459-1484, 2017.

[36] C. Zagaris, H. Park, J. Virgili-Llop, R. Zappulla, M. Romano, and I. Kolmanovsky, "Model predictive control of spacecraft relative motion with convexified keep-out-zone constraints," J. Guid. Control Dyn., vol. 41, no. 9, pp. 2054-2062, 2018.

[37] A. Domahidi, E. Chu, and S. Boyd, "ECOS: An SOCP solver for embedded systems," in Proc. Eur. Control Conf. IEEE, 2013, pp. 30713076.

[38] I. Dunning, J. Huchette, and M. Lubin, "JuMP: A modeling language for mathematical optimization," SIAM Rev., vol. 59, no. 2, pp. 295-320, 2017. 